\title{
Traumatic complete transection of dorsal spinal cord un-associated with spinal fracture or subluxation. Management review
}

\author{
Guru Dutta Satyarthee, Satyajit Panda \\ * Department of Neurosurgery, Neurosciences Centre, AlIMS New \\ Delhi, INDIA
}

\begin{abstract}
Complete transection of spinal cord is an extremely rare occurrence, and usually associated with spinal instability, fracture or spondylolisthesis and also have associated systemic polytrauma involving multiple organs injury. However, a complete transaction of spinal cord unassociated with fracture or dislocation or without abnormality on X-ray and CT scan imaging is extremely uncommon. Kalfas et al. observed $11.2 \%$ of cases had cord transection in the study of sixty-two cases spinal cord-injured patients, who underwent spinal MRI, were additionally had evidence vertebral injury. In 2010, Cha et al reported the first case of transection of cord in the cervical region in a 34-year- adult male, as the first case in literature, which was not associated with any vertebral body fracture or dislocation. Authors report an interesting case, 15-year boy, who had complete transection of the spinal cord associated with polytrauma following a motor vehicle accident, spinal X-ray and CT scan imaging was unremarkable, however spinal MRI scan established the diagnosis. Briefly, imaging, management and pertinent literature are reviewed.
\end{abstract}

\section{INTRODUCTION}

Acute spinal injury can present with a spectrum of pathology including vertebral body fracture, dislocation, cord contusion, but spinal cord transection represents very uncommon occurrence. ${ }^{[1-8]}$ However mostly such cord transection is reported with spinal column injury in form of fracture, dislocation or spondyloptosis. However, unassociated with fracture dislocation is uncommon. In 2010, Cha et al was first to report a case of cervical cord transection of cord in a 34- year- male, which was not associated with fracture or dislocation. ${ }^{[1,2]}$ However, in a detailed radiological review, Kalfas et al. reported his observation of sixty-two spinal cord-injured patients, who underwent spinal MRI evaluation within the first thirty six hours of sustaining spinal injury, seven cases had evidence of anatomical cord transection with various types of fracture and dislocations. ${ }^{[3]}$ Spinal cord injury is usually associated with severe traumatic injury involving spine and other body

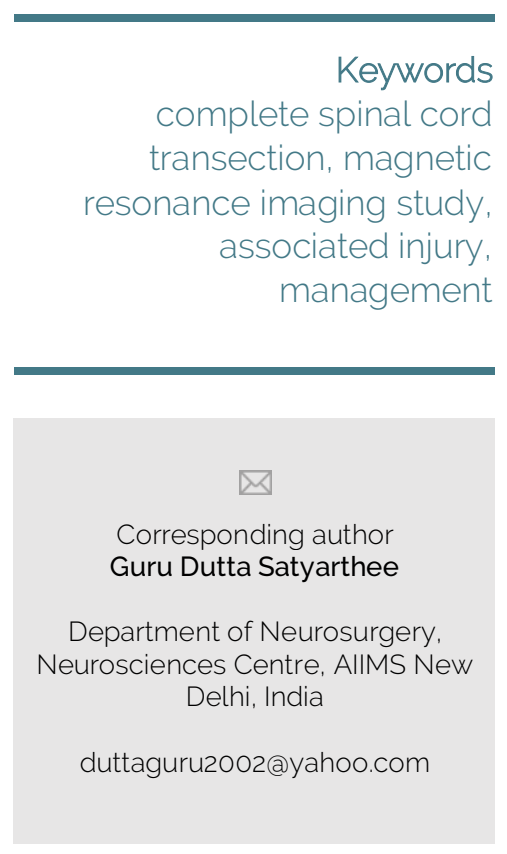

Copyright and usage. This is an Open Access article, distributed under the terms of the Creative Commons Attribution Non-Commercial No Derivatives License (https://creativecommons .org/licenses/by-nc-nd/4.0/) which permits noncommercial re-use, distribution, and reproduction in any medium, provided the original work is unaltered and is properly cited.

The written permission of the Romanian Society of Neurosurgery must be obtained for commercial re-use or in order to create a derivative work.

ISSN online 2344-4959

(C) Romanian Society of Neurosurgery

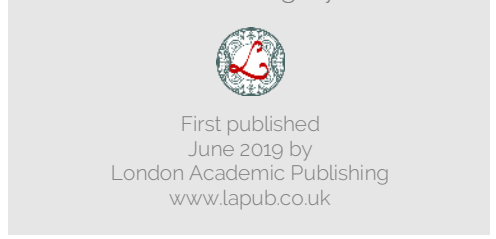


organs. However, paediatric population can sustain various grades of Spinal cord injury without evidence of a radiographic abnormality, and acronym SCIWORA (spinal cord Injury without radiographic abnormality) was coined in the year 1982 by Pang and Wilberger for describing cases sustaining spinal cord injury and presenting with clinical symptoms of traumatic myelopathy, however, no radiographic or computed tomographic scan shows evidence of spinal fracture or instability. [1] SCIWORA is considered rare in adults, but relatively commoner in children. In the paediatric cases, SCIWORA represents about 8 to $19 \%$ of all paediatric spinal cord injuries ${ }^{[4]}$. However, MRI can show presence of in cases of SCIWORA. SCIWORA lesions have predilection for cervical spine commonly but can affect the thoracic or lumbar spine although much less frequently. SCIWORA in adults is rare, but is of considerable important because of the potential problems of management inherent in the diagnosis. Authors report a case, who sustained complete transaction of spinal cord but without association of vertebral column fracture or dislocation. Kalfas et al. reported observation of sixty-two spinal cord-injured patients, who underwent spinal MRI evaluation within the first thirty-six hours of sustaining injury, showed presence of anatomical cord transection in 7 cases. $^{[3]}$

\section{CASE ILLUSTRATION}

A 15- year old male pillion rider presented to emergency services with the complaints of inability to move associate with numbness involving both lower limbs following a high-speed road traffic accident. He wore helmet and received initial first aid was given at primary health centre, evaluation on arrival to our emergency services, he had history of loss of consciousness for ten minutes, when regained consciousness, complained of severe pain in the abdomen. On arrival at hospital his pulse rate was 160 per minute and blood pressure was 70/40/mm Hg. Immediately resuscitation started with colloid and blood transfusion started, with momentary recovery of blood pressure followed by fall. GCS was E3VTM5 and pupils were normal size briskly reacting to light with motor evaluation showed flaccid paraplegia with absent deep tendon jerks.

The $\mathrm{X}$-ray chest and cervical spine was normal. Non-contrast computed tomography of cervical spine showed was essentially normal with no evidence of fracture, subluxation or compromise of spinal canal. (FIGURE 1) cranial computed tomography scan head showed presence of multiple contusions with diffuse cerebral edema with absence of midline shift. Ultrasound abdomen revealed presence of hemoperitoneum, CT scan abdomen revealed presence of hepatic tear with hemoperitoneum.

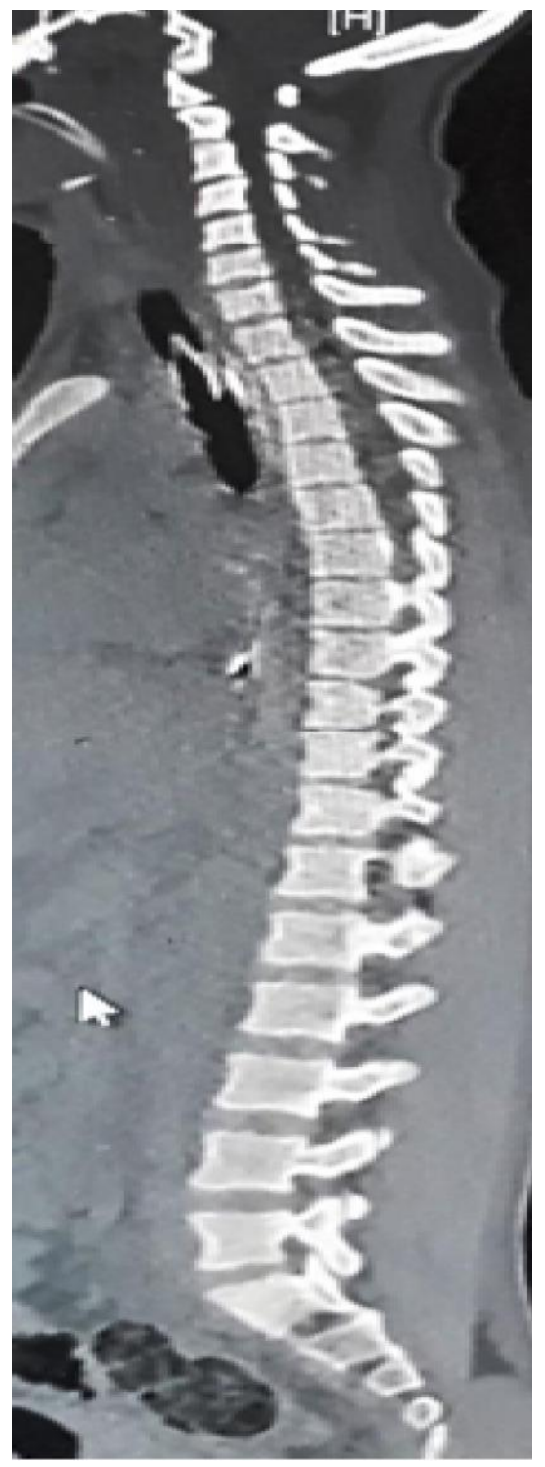

FIGURE 1.

Computed tomography scan spine of 15-year male, sagittal section image of spine showing no evidence of fracture or dislocation.

He was planned for emergency surgical exploration by trauma surgery team. He suffered cardiac arrest in casualty while shifting to operation theatre, but revived and immediately shifted to operation theatre and underwent emergency laparotomy under general anaesthesia. A midline laparotomy done; two litters of blood mixed collection were drained. Intraoperative, solid organs were examined, showed presence of grade -3 liver laceration on anterolateral 
surface in the second segment with bile staining with serosal tear found on greater curvature of stomach and associate retroperitoneal hematoma in ascending and descending mesocolon and gastric tear was also repaired, sub hepatic drain was placed and mesh laparostomy was carried out.

In the post- operative phase, MRI of whole spine was carried out to causes of paraparesis, showed non visualization of the spinal cord from upper

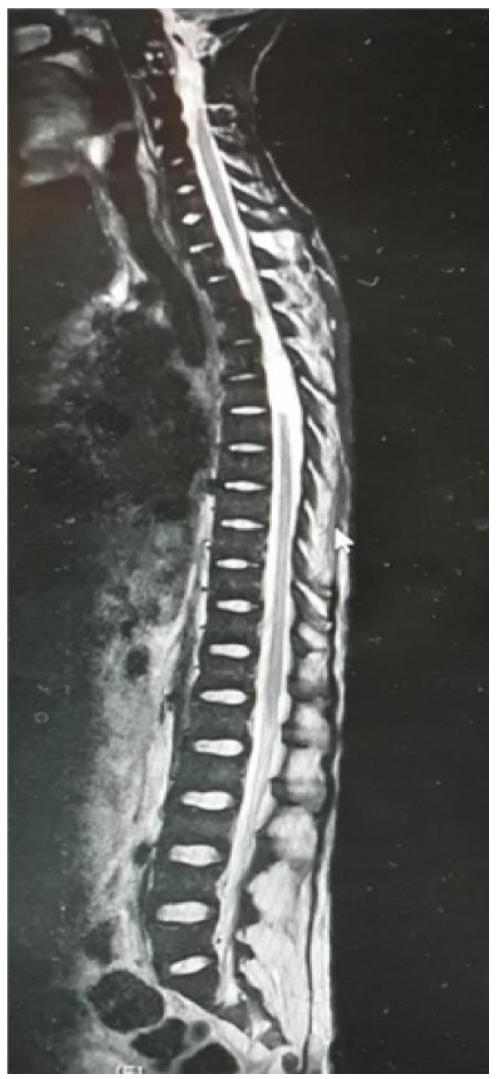

FIGURE 2. MRI, spine, T2 weighted image, sagittal section image of spine showing discontinuity of dorsal spinal cord at level of D3 to lower border of D5 vertebra with retraction of upper and lower cord segment suggestive of complete cord transaction. Foci of blooming seen in the retracted upper cord at D3 level suggestive of haemorrhage.

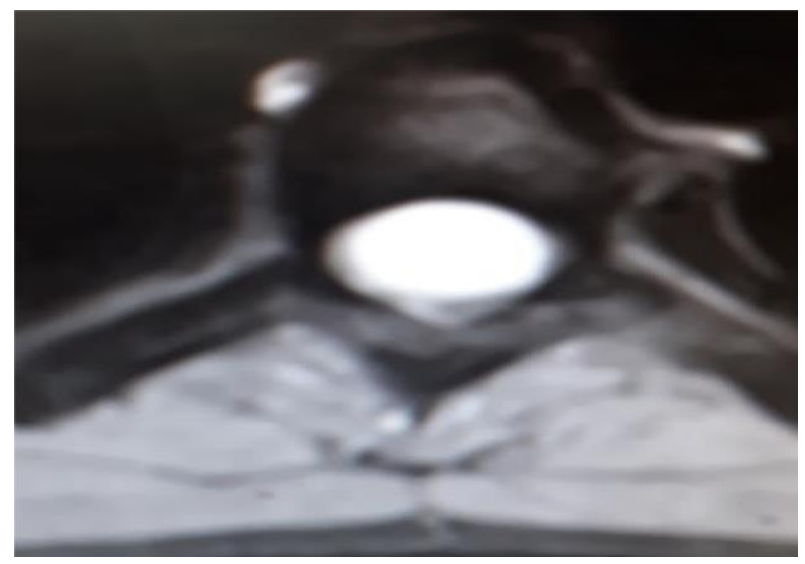

FIGURE 3. Magnetic resonance imaging study of dorsal spine, axial section, $\mathrm{T} 2$ weighted image of showing absence of spinal cord signal. border of D3 to lower border of D5 vertebra with retraction of upper and lower cord segment suggestive of complete cord transaction. (FIGURE 2) Foci of blooming seen in the retracted upper cord at D3 level suggestive of hemorrhage. In the proximal part of the transacted cord there was a linear T2 hyperintensity at D1-D3 level might be wallerian degeneration. T2/STIR hyperintensity seen in the supraspinous and interspinous ligaments from C7 to D10 levels suggestive of sprain in ligaments.

\section{DISCUSSION}

The incidence of SCIWORA is considered highest among children below eight years also being predisposed by presence of weaker neck muscles, and relatively heavy head and more horizontal orientation of facet joints and associated greater elasticity of vertebral ligaments. The cervical spinal cord is most commonly involved [5]. SCIWORA involvement in the thoracic is rare and contributes for about 10 to $13 \%$ of all paediatric SCIWORA cases. ${ }^{[4,5]}$ The commonest cause of thoracic SCIWORA is high velocity trauma, including motor vehicular highspeed vehicular collisions, severe distraction injuries related to lap belts and crush injuries in slow-moving motor vehicles ${ }^{[5]}$ other includes sports injuries, assault or gunshot injury and falls.

In most cases, SCIWORA occurs as a result of hyperextension forces or from a direct frontal impact to the face and May to occur during sports such as diving, wrestling, and baseball. However, Cha et al put forward combined hyperflexion-hyperextension mechanism for cervical cord transection. ${ }^{[2]}$ In children, such movement of the vertebral column allows the musculoskeletal system to move beyond the normal physiological range of motion without the risk of fracture, however injury to the spinal cord is caused contusion or ischemia due to temporary occlusion of vertebral arteries followed by a spontaneous return of vertebrae to their original Venous congestion within the compressed spinal cord is also a pathogenic factor. The onset of clinical symptoms is delayed from a few minutes to 48 hours after injury in about $50 \%$ of patients. This latency is associated with repeated micro-insults to the spinal cord from striking against the unstable vertebrae. Various spinal cord pathology includes, signal cord swelling, contusion or oedema, however cord transaction is uncommon. ${ }^{[5]}$

Patients may present with variety of clinical 
presentation depending on severity of injury and location of involvement of spinal cord includes transient weakness, paraesthesia, numbness, paraparesis, quadriplegia, complete cord transection syndrome and Brown-Sequard syndrome. ${ }^{[17]}$

CT scan spine is best for assessing the associated bony injuries which may need concomitant treatment consideration but does not assess the cord itself. MRI is considered as investigation of choice and is often performed acute phase in the context of recent traumatic spinal injury and picks up spinal cord pathology, associated extramural hematoma, or hematomyelia, or ligament injury, which that may or may not be confirmed by trauma $\mathrm{CT}$. The role of acute MRI is usually to assess for the presence of acute pathologies treatable such as an epidural haematoma or cord compression, which can be targeted by surgical decompression. The identification of significant cord trans section can potentially contraindicate surgery. Kalfas et al analyzed cases had spectrum of lesions including anatomical spinal cord transection in seven cases, other finding were 28 cases had spinal cord deformity secondary to extrinsic compression, 21 had focal cord enlargement/swelling, hyperintensity intramedullary lesions noted in 17 cases, and rest two cases had disc herniations. ${ }^{[3]}$

Boese et al. analyzed 114 patients, on admission, neurologic deficit assessed by the American Spinal Injury Association impairment scale was an in 28\%, B in $17 \%, C$ in $31 \%$, and $D$ in $25 \%$. On basis of MRI, they categorize finding. Type I noted in $43 \%$ cases with no MRI abnormalities and 57\% exhibited abnormal scan results (Type II): $6 \%$ revealed extra neural (Type Imia), $38 \%$ intramural (Type Ibis) and 13\% combined abnormalities (Type Sic). ${ }^{[15]}$

Pang classified MRI finding into five common patterns, first type is complete cord disruption represents most severe injury predominantly involving upper cervical and thoracic spine in younger children as a result of flexion injury. second being major cord haemorrhage with axial MRI showing haemorrhagic involvement of greater than $50 \%$ of cord, next is a minor cord haemorrhage, fourth is isolated cord edema and fifth pattern is groups of patients presenting with acute clinical neurologic deficit consistent with SCIWORA and with no evidence of injury in the cord on MRI study. ${ }^{[17]}$

Prognosis depends on spinal cord pathology and associated spinal injury. For isolated spinal cord injury case with only cord oedema have most favorable prognosis but intermediate for cord oedema and contusion and remains worse for cord contusion and associated intramedullary hemorrhage. The prognosis for spinal cord transaction still remains very poor. However, in cases with SCIWORA, associated extrinsic compression caused by vertebral body fragment and disc must be surgically corrected. In cases with fracture may need spinal fixation and fusion. Management of spinal cord transection is still a mystery, and no clear solution seems in the pipeline in near future. ${ }^{[6-9]}$

Satyarthee advocated ideally care management acute traumatic spinal cord injury should commence immediately at the moment of traumatic impact, continue during safe evacuation, immediate resuscitation by trained paramedics, adequate immobilization, and proper prehospital care, and emergence transportation to a specialized hospital after prompt resuscitation, a detailed neurologic evaluation, pertinent neuroimaging studies, medical intervention including maintenance of airway and circulation, augmentation and maintenance of target level mean arterial pressure, avoidance of any hypotensive episodes, assessment and appropriate care of associated systemic injury, early surgical spinal decompression as judiciously needed. [8] However, these are effective in minor cord injury, but for complete teransection of spinal cord, situation remains gloomy. ${ }^{[8-10]}$

Tabakow et al observed management of cases suffering complete spinal cord injury remains an unsolved clinical problem because of the lack of spontaneous regeneration of injured central axons. ${ }^{[13]}$ Exact management is still not available. [10-16] Various suggested method are suggested mostly on experimental basis. Olfactory ensheathing cells support axon outgrowth in the olfactory system, and transplantaion after spinal cord injury lead to axon regeneration and improvement in recovery. Thornton et al. observed rats with mid-thoracic transection and olfactory ensheathing cells transplantation, and for assessing connectivity across the transection, pseudorabies virus injected into muscles of hind-limb and finally noted presence of large number of serotonergic axons crossed the rostral scar border and the area of neurofilamentpositive axons in the injury site. ${ }^{[11]}$ Authors concluded olfactory ensheathing cells transplantation can increase axonal growth across 
the injury site and may promote recovery of propioception circuit.

Tabakow et al reported 38-year-old with ASIA A injury at 21 months. The olfactory bulbs were removed to obtain a culture containing olfactory ensheathing cells and olfactory nerve fibroblasts. The cultured cells were transplanted following resection of the glial scar. He improved to ASIA C with improved trunk stability, partial recovery of the voluntary movements of the lower extremities, and an increase of the muscle mass in the left thigh. Neuroimaging confirmed grafts producing bridge over left side of the spinal cord with majority of the nerve grafts implant, and further neurophysiological examinations confirmed the restitution of the integrity of the corticospinal tracts. ${ }^{[13]}$

\section{CONCLUSION}

Every cases with suspected head injury sustaining can presenting with shock and paraplegia, a high index of suspicion for cord transaction should also be considered, although rarer. It is highly imperative that paediatrician, neurosurgeons, orthopaedician and emergency physician should be aware for entity total spinal cord transection.

\section{REFERENCES}

1. Pang D, Wilberger JE Jr. Spinal cord injury without radiographic abnormalities in children. J Neurosurg 1982; 57(1):114-129.

2. Cha $\mathrm{YH}$, Cho TH, Suh JK. Traumatic cervical cord transection without facet dislocations--a proposal of combined hyperflexion-hyperextension mechanism: a case report. J Korean Med Sci. 2010 Aug;25(8):1247-50.

3. Kalfas I, Wilberger J, Goldberg A, Prostko ER. Magnetic resonance imaging in acute spinal cord trauma. Neurosurgery. 1988 Sep;23(3):295-9.

4. Carroll T, Smith CD, Liu X et al Spinal cord injuries without radiologic abnormality in children: a systematic review. Spinal Cord 2015; 53(12):842-848.

5. Parizel PM, van der Zijden T, Gaudino S et al. Trauma of the spine and spinal cord: imaging strategies. Eur Spine J. 2010;19 Suppl 1 (S1): S8-17.

6. Satyarthee GD, Sangani M, Sinha S, Agrawal D. Management and Outcome Analysis of Pediatric Unstable Thoracolumbar Spine Injury: Large Surgical Series with Literature Review. J Pediatr Neurosci. 2017 Jul-Sep; 12(3):209-214. Doi: 10.4103/Jpn
7. Farrell CA, Hannon M, Lee LK. Pediatric spinal cord injury without radiographic abnormality in the era of advanced imaging. Curr Opin Pediatr. 2017 ;29(3):286-290.

8. Satyarthee GD. Ways to Improve Outcomes of Traumatic Acute Spinal Cord Injury: Integrated Approaches of Improved Prehospital Care, the Adoption of Synergistic Medical and Surgical Intervention, Along with Care for Associated Systemic Injury and Rehabilitation and Social Inclusion. World Neurosurg. 2017;101:786-787

9. Lukovic D, Moreno-Manzano V, Lopez-Mocholi E, Rodriguez-Jiménez FJ, Jendelova $P$, Sykova $E$, Oria $M$, Stojkovic M, Complete rat spinal cord transection as a faithful model of spinal cord injury for translational cell transplantation. Erceg S. Sci Rep. 2015 Apr 10;5:9640.

10. Verma SK, Singh PK, Agrawal D, Sinha S, Gupta D, Satyarthee GD, Sharma BS. O-arm with navigation versus C-arm: a review of screw placement over 3 years at a major trauma center. Br J Neurosurg. 2016 Dec;30(6):658-661.

11. Thornton MA, Mehta MD, Morad TT, Ingraham KL , Khankan RR, Griffis KG ,et al. Evidence of axon connectivity across a spinal cord transection in rats treated with epidural stimulation and motor training combined with olfactory ensheathing cell transplantation. Exp Neurol. 2018 Jul 26. pii: S0014-4886(18)30263-2. doi: 10.1016/j.expneurol.2018.07.015.

12. Ahmann PA, Smith SA, Schwartz JF, et al. Spinal cord infarction due to minor trauma in children. Neurology. 1975;25:301-7.

13. Tabakow P, Raisman G, Fortuna W, Czyz M, Huber J, Li D, Szewczyk $P$, et al. Functional regeneration of supraspinal connections in a patient with transected spinal cordfollowing transplantation of bulbar olfactory ensheathing cells with peripheral nerve bridging. Cell Transplant. 2014;23(12):1631-55.

14. Chandrashekhara SH, Kumar A, Gamanagatti S, Kapoor K, Mukund A, Aggarwal D, Sinha S. Unusual traumatic spondyloptosis causing complete transection of spinal cord. Unusual traumatic spondyloptosis causing complete transection of spinal cord. Int Orthop. 2011 Nov;35(11):1671-5.

15. Boese CK, Oppermann J, Siewe J, Eysel P, Scheyerer MJ, Lechler P. Spinal cord injury without radiologic abnormality in children: a systematic review and meta-analysis. J Trauma Acute Care Surg. 2015 Apr;78(4):874-82.

16. Szwedowski D, Walecki J Spinal cord injury without radiographic abnormality (SCIWORA)—clinical and radiological aspects. PolJ Radiol. 2014; 79:461-464.

17. Pang D. Spinal cord injury without radiographic abnormality in children, 2 decades later. Neurosurgery 2004; 55:1325-1342. 